\title{
Manejo docente de las tecnologías de la información y comunicación. Cuenca, Ecuador
}

\section{Teaching management of information and communication technologies. Cuenca, Ecuador}

Neli Norma Gonzales Prado

César Augusto Trelles Zambrano

Janeth Catalina Mora Oleas

Universidad de Cuenca, Ecuador

Autor por Correspondencia: neli.gonzales@ucuenca.edu.ec, cesar.trellesz@ucuenca.edu.ec, catimorao@yahoo.es

Fecha de recepción: 31 de Enero de 2017 - Fecha de aceptación: 25 de Marzo de 2017

Resumen: Este trabajo presenta resultados de la percepción docente sobre el uso de las Tecnologías de la Información y Comunicación (TIC), finalidad, frecuencia, nivel de dominio, impacto y disponibilidad para su implementación en el proceso enseñanza-aprendizaje. Participaron 365 docentes, que laboran en instituciones de Educación General Básica (EGB) Superior y Bachillerato General Unificado (BGU), seleccionados por métodos probabilísticos. Se elaboró un cuestionario que incluyó ítems de las cinco áreas contempladas en el marco conceptual TIC en Educación, del Banco Interamericano de Desarrollo (BID). Los resultados evidenciaron que los recursos más utilizados son: procesador de texto, hoja de cálculo, internet y presentaciones. Dichos recursos se emplean fundamentalmente para la gestión docente, con menor frecuencia para el aprendizaje asistido y escasamente para promover el aprendizaje autónomo del estudiante. En conclusión, los docentes priorizan el uso de las TIC en la planificación formal y como apoyo de clase, aún es necesario promover su incorporación a la gestión del aprendizaje.

Palabras clave: TIC; gestión docente; aprendizaje asistido; aprendizaje autónomo

Abstract: This paper presents teacher's perception results on computer resources used, the purpose and frequency of use; level of mastery; impact and their availability for implementation. Participants were 365 teachers who work in General Basic Higher Education and Unified General Baccalaureate institutions. A questionnaire was elaborated that included items from the five areas of the ICT conceptual framework in Education of the Inter-American Development Bank was used. Results showed that the most used resources are: word processor, spreadsheet, internet and presentations. These resources are mainly used for teaching management, less frequently for assisted learning and scarcely to promote student autonomous learning. In conclusion, teachers prioritize the use of ICT in formal planning and as class support, it is still necessary to promote their incorporation into the management of learning.

Key words: TIC; teaching management; assisted learning; autonomous learning 


\section{Introducción}

Las Tecnologías de la Información y Comunicación (TIC), pueden ser definidas como el conjunto de "tecnologías para el almacenamiento, recuperación, proceso y comunicación de la información" (Belloch, 2012). En la actualidad las TIC están incidiendo en las diferentes actividades desarrolladas por el ser humano, el sector de la educación no es la excepción y cada vez son más los estudios que tratan de aportar para que la incorporación de las TIC en los procesos de enseñanza-aprendizaje genere resultados positivos.

Varios son los organismos que aportan al tema de TIC, en 1999 por ejemplo se creó el Instituto de Estadística de la United Nations Educational Scientific and Cultural Organization (UIS) con la finalidad de desarrollar y proveer estadísticas exactas y relevantes, en el contexto de cada país, en los campos de: la educación, la ciencia, la tecnología, la cultura y la comunicación (United Nations Educational Scientific and Cultural Organization [UNESCO], 2013). Por su parte el Plan de Acción para América Latina y el Caribe, considera a las TIC como instrumentos de desarrollo económico y de inclusión social (Comisión Económica para América Latina y el Caribe [CEPAL], 2013). En el área educativa concretamente, diversas instituciones y organizaciones, han ofrecido cursos de capacitación docente para el uso de las TIC, y el desarrollo de apoyos didácticos digitales, entre ellos se encuentran los cursos que ofrece la plataforma tecnológica Coursera que oferta cursos en diferentes áreas del conocimiento de forma virtual y el Portal Educativo de las Américas de la Organización de los Estados Americanos.

En el Ecuador, se han desarrollado políticas para facilitar la incorporación de las TIC en el área educativa. Para Peñaherrera (2012) las TIC se han integrado mediante dotación de infraestructura, software educativo, equipamiento de aulas con recursos informáticos, capacitación al profesorado, creación de portales educativos, soporte técnico a las escuelas, entre otros. Durante el 2002 se ejecutó el programa maestr@s.com, dirigido a docentes en ejercicio, consistió en la entrega de computadores personales y capacitación en el manejo de suite ofimática. En el 2007, se implementó el plan de las unidades del milenio, específicamente en las zonas rurales, como parte integral de la política gubernamental para mejorar la calidad de la educación pública, incorporando edificaciones con recursos pedagógicos adecuados, equipamiento moderno y tecnología avanzada (Ministerio de Educación [MinEduc], 2007). En el 2014, el Ministerio de Educación entregó kits tecnológicos a docentes de instituciones educativas, de acuerdo a la planificación la meta del proyecto fue integrar a la comunidad educativa en línea a un total de 5.500 instituciones educativas y entregar computadoras portátiles con servicio de conectividad a 147.170 docentes de todo el país (MinEduc, 2014). El Currículo de los niveles de educación obligatoria por su parte fomenta el uso de las TIC en todas las áreas de estudio, por ejemplo en el área de Matemática plantea utilizar los nuevos recursos tecnológicos para favorecer el quehacer matemático (MinEduc, 2016). De igual forma prevé diseñar estrategias para el uso de las TIC en el abordaje de las diferentes áreas de estudio. En los últimos años el Ministerio de Educación mediante su programa de Formación Continua ha venido ofertando cursos de capacitación en las diferentes disciplinas a los docentes del magisterio fiscal, estos cursos han sido planificados para que sean dictados en modalidad semipresencial y virtual con una duración de 330 horas, de las cuales 60 estaban destinadas específicamente a capacitación en el manejo de TIC. 
Con los antecedentes expuestos, es difícil hablar de procesos de enseñanza - aprendizaje, sin pensar en el uso de las TIC, pero no solo como un complemento, sino como la vía de cambio cultural, que permita mejorar la calidad de la educación, ahorrando tiempo en procesos mecánicos y profundizando aspectos relevantes como conceptualizaciones e interpretaciones argumentadas. Al respecto Kumari \& D’Souza (2016) señalan que las TIC usadas apropiadamente, desarrollan la creatividad y habilidades de pensamiento de orden superior en los estudiantes, mismas que son esenciales en el siglo XXI.

Por tanto, la incorporación de las TIC exige una redefinición de los roles de docentes y estudiantes. Los docentes deben convertirse en asesores, colaboradores, y dinamizadores del aprendizaje mediante una acción tutorial, direccionada a seleccionar los programas de formación y diagnosticar sus necesidades y problemas académicos; los estudiantes deben formar parte activa del proceso de enseñanza, a través de la búsqueda, intercambio e interpretación de la información, fomentando su autonomía (Salinas, 2008).

Es importante también indicar que no basta con la dotación de infraestructura tecnológica, es necesario ejecutar políticas que propongan dar un tratamiento integral al uso de las TIC en educación, "la tecnología informática por sí misma no genera aprendizaje de forma espontánea, sino que depende de los fines educativos, de los métodos didácticos y de las actividades que realizan los alumnos con los ordenadores en el aula" (Area, 2007). En este sentido Wengslinky (1998) a través de la prueba National Assessment of Educational Progress (NAEP) en Estados Unidos, concluyó que cuando los profesores estaban lo suficientemente capacitados para dirigir a los estudiantes, el uso de la tecnología parecía estar asociado con mejoras significativas en los logros de aprendizaje en matemáticas.

A pesar que el Ecuador ha realizado importantes propuestas con el propósito de conseguir la integración de las tecnologías a la educación, algunos estudios encontraron que todavía hay mucho por hacer para que esta aspiración se concrete en la práctica (Peñaherrera, 2012; Valdivieso, 2010). Para seguir avanzando en este proceso, es importante determinar cómo en la actualidad las instituciones educativas y sus docentes están incorporando los recursos tecnológicos como parte de los procesos educativos. Con este objetivo se desarrolló la presente investigación de enfoque cuantitativo a nivel descriptivo y relacional.

\section{Acerca de la investigación}

\section{Población y muestra}

De un total de 6934 docentes que laboran en los niveles de EGB superior y BGU en el cantón Cuenca, datos oficiales del Ministerio de Educación, 2014, participaron en la presente investigación 365 docentes: 53\% mujeres y 47\% hombres; con una media de edad de 41.31 años $(\mathrm{DE}=10.5)$ y una media de años de labor docente de 14.8 años (DE =10.4). El tamaño de la muestra se calculó con un nivel de confianza del $95 \%$ y un error muestral del $5 \%$.

La muestra fue seleccionada por método polietápico probabilístico; se inició con la estratificación de los maestros por distritos, por tipo de sostenimiento (fiscal-particular) y por ubicación geográfica (zona urbana o rural). Se eligieron aleatoriamente 20 instituciones, a partir 
de las cuales se obtuvo la muestra de docentes, con afijación proporcional respecto a la población.

\section{Técnicas e instrumentos de recolección de información}

Se empleó la técnica de la encuesta auto aplicada; se utilizó un cuestionario construido ad hoc para el estudio; para el planteamiento de los ítems se tomaron en cuenta las cinco áreas del marco conceptual TIC en educación del BID, relacionadas con: infraestructura, contenidos, recursos humanos, gestión y políticas a partir de las cuales se propusieron cinco secciones: sección A, datos informativos de la institución y el docente (8 ítems). Sección B, aspectos relacionados con el uso de las TIC (5 ítems), más dos escalas tipo Likert; la primera, de 11 ítems relativos a la incorporación de recursos informáticos al proceso educativo en tres dimensiones: el aprendizaje autónomo, el aprendizaje asistido y como medio de comunicación e información. La segunda escala de 8 ítems correspondiente a la utilidad de los recursos en la gestión docente y en el aprendizaje asistido. Las escalas registraron consistencias internas aceptables dadas por un alpha de Cronbach de .79 y de .87 respectivamente. Sección C, relacionada con el dominio en el uso de recursos informáticos ( 2 ítems). Sección D referida a la percepción del impacto del uso de dichos recursos ( 2 ítems) y por último la sección E relacionada a los recursos institucionales disponibles para la implementación de las TIC (4 ítems).

\section{Análisis de datos}

El procesamiento de los datos se realizó con el programa estadístico SPSS versión 22. El análisis univariado se realizó mediante: frecuencias absolutas, frecuencias porcentuales, medidas de tendencia central, variabilidad, posición y forma. Los valores inferenciales se presentaron con intervalos de confianza del 95\%, la comparación de grupos se realizó mediante U Mann de Whitney y el análisis relacional con el coeficiente de correlación de Spearman (rs). Las conclusiones se tomaron considerando un nivel de significancia menor al $5 \%(\mathrm{p}<.05)$.

\section{Resultados}

Del total de docentes participantes el $62.5 \%$ labora en la zona urbana y el $66.3 \%$ en instituciones fiscales. E1 51.2\% son docentes de EGB superior; el 68\% trabaja en jornada matutina. El $63.1 \%$ posee título de tercer nivel en el área de la educación, el 36.9\% tiene título en otras áreas como: Química y Farmacia, Ingeniería en Sistemas, Ingeniería Comercial y Jurisprudencia entre otras. Ver tabla 1.

\section{Tabla 1}

\section{Características de la muestra}

\begin{tabular}{|c|c|c|c|c|c|}
\hline Indicadores & & $\%$ & Indicadores & & $\%$ \\
\hline \multirow[t]{2}{*}{ Ubicación } & zona urbana & 62.5 & \multirow[t]{2}{*}{ Sostenimiento } & Fiscal & 66.3 \\
\hline & zona rural & 37.5 & & Particular & 33.7 \\
\hline \multirow[t]{3}{*}{ Jornada } & Matutina & 68 & \multirow[t]{3}{*}{ Título tercer nivel } & Ciencias de la Educación (C.E) & 25.8 \\
\hline & Vespertina & 28 & & C.E con especialización & 37.3 \\
\hline & Nocturna & 4 & & Otras áreas & 36.9 \\
\hline
\end{tabular}




\begin{tabular}{|c|c|c|c|c|c|}
\hline \multirow[t]{2}{*}{ Nivel en el que labora } & EGB superior & 51.2 & \multirow[t]{2}{*}{ Cuarto nivel } & Con & 20 \\
\hline & Bachillerato & 48.8 & & Sin & 80 \\
\hline
\end{tabular}

Los participantes registraron edades entre 22 y 61 años, IC 95\% [40.2; 42.5], el 25\% de docentes menores a 33 años y el $25 \%$ mayores a 50 años. Respecto a los años de servicio docente se registró un mínimo de 1 año y un máximo de 40, IC 95\% [13.56; 15.83]; el 25\% de docentes con 6 años de servicio y el $25 \%$ con más de 23 años. Las distribuciones de frecuencia de la edad $\mathrm{y}$ de años de labor docente no son distribuciones normales $(\mathrm{K}-\mathrm{S}=1.597 ; \mathrm{p}=.012$ y $\mathrm{K}-\mathrm{S}=2.811$; $\mathrm{p} \square .000$ respectivamente), existe una mayor concentración de participantes jóvenes y profesionales con menos de 10 años de servicio. La media de edad de los docentes de las instituciones particulares $(\mathrm{M}=36.91$ años; $\mathrm{DE}=9.12$ años $)$ es menor $(\mathrm{U}=8182 ; \mathrm{p} \square .000)$ a la media reportada en las instituciones fiscales $(M=43.51$ años; $\mathrm{DE}=10.42$ años). Ver Figura 1. De la misma manera, los años de labor docente registraron una media menor ( $U=9446 ; \mathrm{p} \square$ .000) en las instituciones educativas particulares $(\mathrm{M}=11.21$ años; $\mathrm{DE}=8.13$ años) respecto a los años de labor de los docentes de instituciones fiscales $(M=16.57$ años; $D E=11.04$ años $)$. Ver Figura 2.
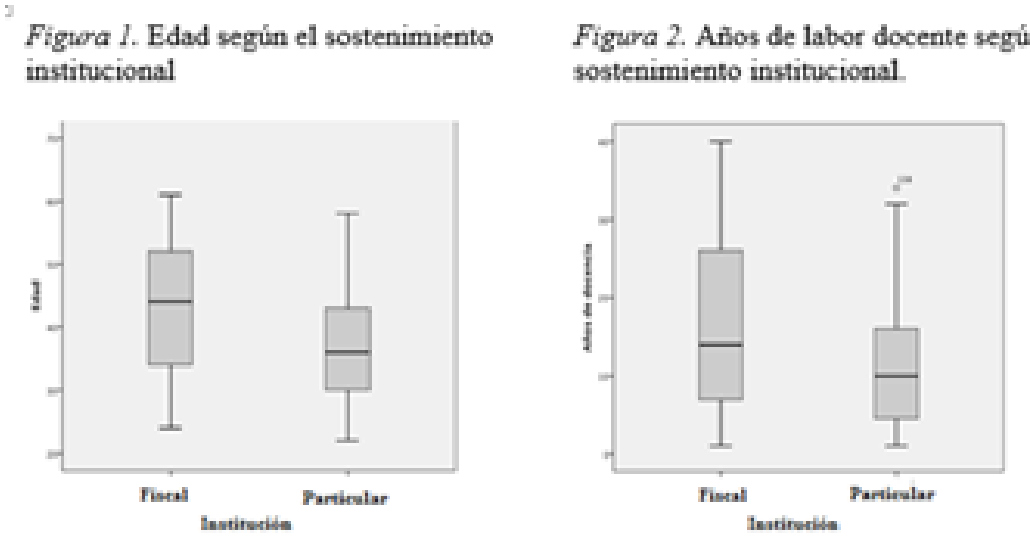

Un $88.2 \%$ de docentes emplea las TIC para la elaboración de material educativo, el 95.6\% utiliza el internet con este fin. El 44.4\% de docentes señaló que semanalmente apoyan sus clases con el uso de recursos informáticos, el 29\% lo hace mensualmente y el $14.8 \%$ lo hace a diario, el $6 \%$ una vez por trimestre, el $7 \%$ una vez al año y un $3.8 \%$ nunca lo hace.

Los recursos que se utilizan con mayor frecuencia son: las presentaciones (53.4\%), los procesadores de texto (47.7\%) y los sitios web (34.8\%). Ver Tabla 2. El porcentaje de no respuestas osciló entre el 18\% y $37 \%$.

Tabla 2

\begin{tabular}{llllll}
\hline \multicolumn{6}{c}{ Frecuencia del uso de recursos informáticos } \\
\hline Recurso & Nunca & Pocas veces & Muchas veces & Siempre & NC \\
\cline { 2 - 6 } & $\%$ & $\%$ & $\%$ & $\%$ & $\%$ \\
\hline Procesador de texto & 11.2 & 14.8 & 19.5 & 28.2 & 26.3 \\
\hline Hoja de cálculo & 17.5 & 16.7 & 18.4 & 17 & 30.4 \\
\hline Presentaciones & 5.8 & 22.7 & 27.9 & 25.5 & 18.1 \\
\hline
\end{tabular}




\begin{tabular}{llllll}
\hline Sitios web & 14.5 & 24.7 & 21.4 & 13.4 & 26 \\
\hline Software educativo & 17.5 & 25.5 & 10.7 & 9.3 & 37 \\
\hline
\end{tabular}

En cuanto a la utilidad de los recursos informáticos, se encontró una media de 3.49 (DE = $0.51)$ para las actividades de gestión docente y una media $3.14(\mathrm{DE}=0.65)$ para las actividades de aprendizaje asistido, lo que significa una utilidad media alta en el primer caso y una utilidad media en el segundo caso. Ver tabla 3.

Tabla 3

Utilidad de las TIC en el proceso educativo (escala entre 1 y 4)

\begin{tabular}{llllll}
\hline Actividades de gestión docente & $\mathbf{M}$ & $\mathbf{D E}$ & Actividades de Aprendizaje Asistido & $\mathbf{M}$ & $\mathbf{D E}$ \\
Elaborar planificaciones curriculares & 3.49 & 0.67 & Apoyar el desarrollo de la clase & 3.36 & 0.70 \\
Elaborar guías de trabajo & 3.38 & 0.70 & Fomentar el debate & 2.91 & 0.82 \\
Buscar información & 3.66 & 0.53 & Fortalecer el trabajo en grupo & 3.04 & 0.88 \\
Elaborar actividades de evaluación & 3.46 & 0.68 & Incentivar la investigación estudiantil & 3.30 & 0.75 \\
Total & 3.49 & 0.51 & Total & 3.14 & 0.65 \\
\hline
\end{tabular}

Los docentes consultados consideran que en general su nivel de dominio en el manejo de recursos informáticos es muy bueno: $\mathrm{M}=3.07$ ( $\mathrm{DT}=0.68$ ); el mayor nivel de dominio corresponde al uso del correo electrónico, en tanto que los menores niveles de dominio se reportaron en el manejo de hojas de cálculo y videos a los que califican como buenos. Ver Tabla 4.

Tabla 4

Nivel de dominio en el manejo de recursos informáticos

\begin{tabular}{lll}
\hline & M & DE \\
\hline Procesador de texto & 3.26 & 0.76 \\
\hline Hoja de cálculo & 2.82 & 0.89 \\
\hline Presentaciones power point & 3.17 & 0.82 \\
\hline Búsqueda de información en la red & 3.33 & 0.72 \\
\hline Dispositivos extraíbles & 3.16 & 0.83 \\
\hline Redes sociales & 3.01 & 0.94 \\
\hline Correo electrónico & 3.45 & 0.69 \\
\hline Elaboración de videos & 2.34 & 1.11 \\
\hline
\end{tabular}

Los docentes que laboran en instituciones particulares reportaron un mayor nivel de dominio de los recursos informáticos, comparados con el reportado por los docentes que laboran en las instituciones fiscales $(\mathrm{U}=9446 ; \mathrm{p}=.000)$. Los primeros alcanzaron una media de 3.24 $(\mathrm{DT}=0.59)$ que corresponde a un nivel de dominio muy bueno, los docentes fiscales por su parte alcanzaron una media de 2.97 (DT = 0.71) que corresponde a buen nivel de dominio. También se encontró una relación inversa moderada entre el nivel de dominio y la edad $(r s=-.4 ; p \square .000)$ y entre el nivel de dominio de recursos informáticos y los años de labor docente ( $r s=-.311 ; p$ $.000)$. 
El $60.5 \%$ de los docentes consultados mencionan que se capacitaron por sí mismos en el uso de las TIC, en segundo lugar, ubican los cursos de capacitación con un 55.3\%, seguido de la capacitación informal con 45.2\%. Ver Figura 3.

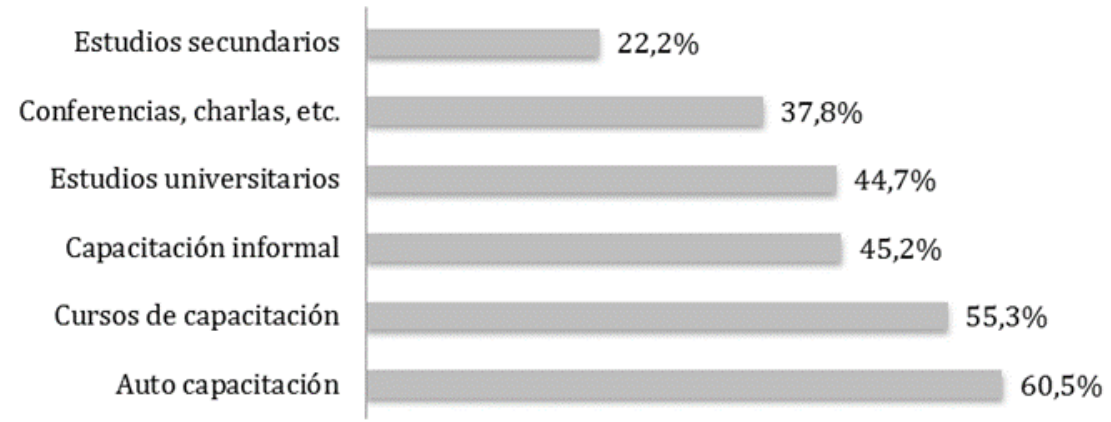

Figura 3. Espacios de capacitación en el uso de las TIC

Más del 75\% de los docentes consideran que el aporte de los recursos informáticos en el proceso educativo es efectivo y significativo, sin embargo un $26.3 \%$ señala que el impacto de los recursos informáticos en el fortalecimiento de la capacidad de cálculo mental de los estudiantes es insignificante o débil, del mismo modo el $54.2 \%$ considera que los recursos informáticos no contribuyen de manera efectiva o significativa en la promoción de la honestidad académica de los estudiantes. Ver Tabla 5.

Tabla 5

\begin{tabular}{|c|c|c|c|c|c|}
\hline \multicolumn{6}{|c|}{ Percepción del impacto del uso de las TIC } \\
\hline & Insignificante & $\begin{array}{l}\text { D } \\
\text { éb } \\
\text { il }\end{array}$ & $\begin{array}{l}\text { Efectiv } \\
\text { o }\end{array}$ & $\begin{array}{l}\text { Significativ } \\
\text { o }\end{array}$ & NC \\
\hline & $\%$ & $\%$ & $\%$ & $\%$ & $\%$ \\
\hline Motivan la adquisición de nuevos conocimientos & 1.1 & 9.9 & 39.8 & 44.9 & 4.4 \\
\hline Mejoran la captación de conceptos en los estudiantes & 0.8 & 9.3 & 49.6 & 34.2 & 6 \\
\hline Fortalecen el cálculo mental del estudiante & 5.8 & 20.5 & 37.5 & 23.8 & $\begin{array}{l}12 . \\
3\end{array}$ \\
\hline Promueven la honestidad académica & 17.5 & 36.7 & 21.1 & 15.9 & 8.8 \\
\hline Mejoran las presentaciones académicas & 2.5 & 15.3 & 41.1 & 33.2 & 7.9 \\
\hline Contribuyen a ampliar los conocimientos del docente & 0.5 & 3.3 & 36.4 & 54.2 & 5.5 \\
\hline Promueven una constante capacitación del docente & 0.3 & 5.2 & 35.1 & 50.1 & 9.3 \\
\hline Agilitan la planificación de las clases & 1.6 & 8.2 & 46.8 & 37 & 6.3 \\
\hline Facilitan la explicación de procesos de razonamiento & 1.9 & 12.3 & 47.1 & 29 & 9.6 \\
\hline Facilitan la elaboración de actividades de evaluación & 1.4 & 12.1 & 47.1 & 32.6 & 6.8 \\
\hline Presentan información confiable para el docente & 0.8 & 3.6 & 39.2 & 50.7 & 5.8 \\
\hline $\begin{array}{l}\text { Permiten la búsqueda de información con mayor } \\
\text { rapidez }\end{array}$ & 0.8 & 3.6 & 39.2 & 50.7 & 5.8 \\
\hline
\end{tabular}




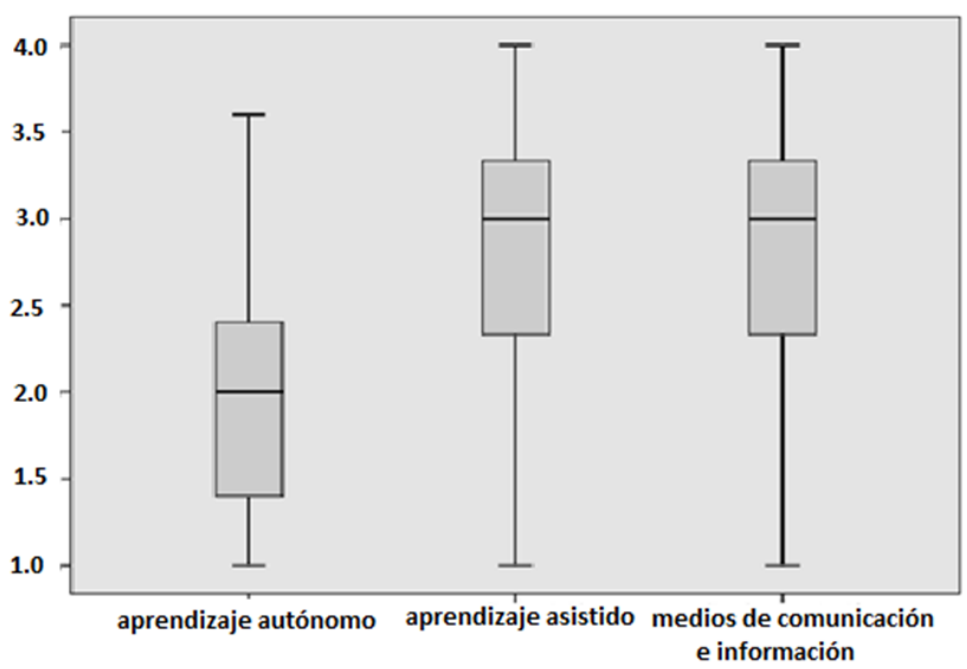

Figura 4. Incorporación de las TIC en el proceso educativo: frecuencia

La incorporación de recursos para el aprendizaje autónomo y como medios de comunicación es similar a nivel de EGB superior y en BGU, sin embargo, la incorporación de los recursos informáticos para el aprendizaje asistido en EGB registró una media de $2.79(\mathrm{DE}=0.69)$ y en BGU una media de 3.02 ( $\mathrm{DE}=0.67)$, diferencia con significancia estadística $(\mathrm{U}=4574 ; \mathrm{p}=$ .008). Por otro lado, la incorporación de los recursos informáticos como recursos para el aprendizaje autónomo, para el aprendizaje asistido y como medios de comunicación e información es similar en: instituciones fiscales y particulares, en instituciones de la zona urbana y rural y no depende del sexo del docente ( $p \square .05)$.

Se encontró una relación inversa, aunque débil entre la incorporación de los recursos informáticos para el aprendizaje asistido y la edad de los docentes ( $r s=-.189 ; \mathrm{p}=.028)$, sin embargo, no se registraron relaciones entre la incorporación de los recursos informáticos para el aprendizaje autónomo y la edad de los docentes o entre la incorporación de los recursos informáticos como medios de comunicación y la edad de los docentes. Tampoco se encontró relación entre la incorporación de los recursos informáticos y los años de labor docente ( $\mathrm{p} \square$ $.05)$.

En relación a los recursos disponibles, en instituciones fiscales y particulares, para la implementación de las TIC, se encontraron similitudes en cuanto a la dotación de laboratorios informáticos, en capacitación en el uso de las TIC y asignación de personal docente especializado en el uso de recursos informáticos. Sin embargo, la adquisición de equipos tecnológicos es mayor en las instituciones particulares $(\mathrm{X} 2=17.536 ; \mathrm{p}<.000)$, así como la adquisición de repuestos $(\mathrm{X} 2=24.84 ; \mathrm{p}<.000)$. Ver Figura 5. 


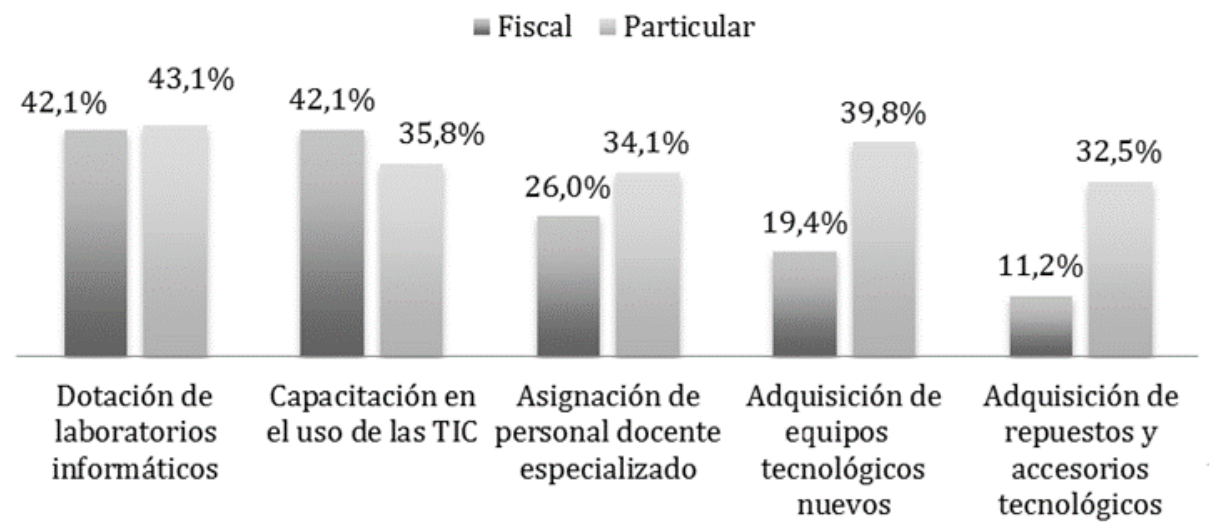

Figura 5. Recursos institucionales para la implementación de las TIC

El 47.6\% de docentes califican como muy bueno u óptimo el acceso a las computadoras en las instituciones, el 39.2\% califica de igual forma el acceso a los proyectores y a la sala de audiovisuales y el 38,6\% el acceso a la Internet. Por otra parte, aproximadamente el 50\% de los docentes califica como deficiente el acceso a: reproductoras y grabadoras de audio y video, cámaras fotográficas, escáner, pizarras digitales y aulas equipadas con proyectores. Ver Tabla 6.

\section{Tabla 6}

Nivel de acceso a los recursos informáticos en la institución

\begin{tabular}{lllll}
\hline \multirow{2}{*}{ Recursos Informáticos } & \multicolumn{2}{l}{ Nivel de Acceso } & \\
\cline { 2 - 5 } & Deficiente & Bueno & Muy Bueno & Óptimo \\
\hline Reproductoras y grabadoras de video & 49.0 & 25.3 & 14.9 & 10.8 \\
\hline Reproductoras y grabadoras de audio & 46.6 & 25.7 & 18.5 & 9.2 \\
\hline Cámaras fotográficas & 61.5 & 18.5 & 13.3 & 6.7 \\
\hline Escáner & 63.7 & 17.2 & 12.2 & 6.9 \\
\hline Pizarras digitales & 65.3 & 14.9 & 11.2 & 8.6 \\
\hline Computadoras & 16.5 & 35.9 & 29.3 & 18.3 \\
\hline Internet & 32.4 & 28.9 & 22.4 & 16.2 \\
\hline Proyectores móviles & 27.4 & 33.4 & 20.8 & 18.4 \\
\hline Sala de Audiovisuales & 26.2 & 34.6 & 18.5 & 20.7 \\
\hline Aulas equipadas con proyectores & 46.0 & 22.8 & 13.8 & 17.4 \\
\hline
\end{tabular}

\section{Discusión y conclusiones}

En Cuenca, cerca del $90 \%$ de los docentes consultados mencionó utilizar las TIC y el internet para elaborar material de trabajo en el aula, además el 44.4\% señaló que semanalmente usa recursos informáticos como apoyo en sus clases; estos valores implican una importante incorporación de los recursos informáticos, particularmente el uso del internet, en las actividades educativas. En este aspecto, el comportamiento de los docentes no difiere de la población en general; según el Instituto Nacional de Estadísticas y Censos (INEC, 2015), el 65.6 \% de la población del Ecuador que usa internet, lo ha hecho por lo menos una vez al día en los últimos 12 meses. 
El informe regional de la integración de las TIC en la educación y de la aptitud digital, 2010, señala que en el Ecuador no existen docentes: calificados en habilidades básicas de TIC, capacitados en enseñar materias utilizando TIC, ni que se encuentren enseñando materias utilizando recursos TIC; apenas un $4 \%$ de docentes enseñaban habilidades básicas de computación e informática (UNESCO, 2013). En el presente estudio el nivel de dominio en el manejo de recursos fue autoevaluado como muy bueno y bueno, específicamente en: el uso de correos electrónicos, búsqueda de información en la red, procesador de texto y presentaciones power point; en este sentido, se podría asumir que se ha logrado un avance en las habilidades de los docentes en el manejo de las TIC. Se encontró una percepción de mayor dominio en docentes jóvenes, dato que coincide con los expuestos en el informe de Tecnologías de Información y Comunicación TIC (INEC, 2015) según el cual el porcentaje de personas que usan computadora disminuye linealmente a medida que aumenta la edad: del grupo de 16 a 24 años, el $76.1 \%$ la emplean; de 25 a 34 años, el $57.6 \%$, de 35 a 44 años, el 45\%. La edad avanzada se relaciona con las pocas posibilidades de habilidades motoras para el uso de una computadora, como describe una investigación sobre las concepciones docentes acerca del uso de las TIC en educación secundaria y los cambios que generan en dichas concepciones cuando los docentes son expuestos a experiencias de interacción con herramientas web 2.0 (Chiappe, Mesa, \& Álvarez, 2013).

Según los docentes, utilizan las TIC en primer lugar, para actividades de gestión y en segundo para actividades de aprendizaje asistido; ya que las actividades de gestión involucran trabajo fuera del aula, convendría conocer desde otra fuente la percepción de la intensidad del uso docente de estos recursos. El 75\% de docentes señala que las TIC impactan significativamente en el proceso educativo, especialmente en la ampliación de conocimientos y en tareas de planificación docente para la enseñanza. Esto concuerda con el estudio desarrollado en Andalucía por Boza, Toscano \& Méndez (2009) que concluyen que las TIC se utilizan fundamentalmente para buscar información y reforzar aprendizajes, pero consideran que sigue sin aprovecharse el potencial comunicativo y de trabajo cooperativo entre los estudiantes.

En cuanto a la dotación de recursos se encontraron similitudes tanto en instituciones fiscales como particulares, sin embargo, existen diferencias significativas a favor de los colegios particulares en cuanto a: asignación de personal docente especializado, adquisición de equipos tecnológicos y repuestos y accesorios. En relación al nivel de acceso a los recursos informáticos, los docentes califican entre bueno a óptimo los siguientes recursos: computadoras, internet, proyectores móviles y sala de audiovisuales.

Por su parte en lo que respecta a la capacitación en el uso de TIC los docentes manifiestan que la principal fuente mediante la cual han adquirido conocimientos en el tema es la auto-capacitación, está apreciación coincide con lo detectado en otras ciudades del país. Valdivieso, (2010) encontró que la mayoría de docentes participantes en su estudio no han recibido capacitación formal en TIC, sino que lo han hecho de manera autodidacta por cuenta propia. Es importante entonces coordinar de mejor manera las políticas públicas en materia de capacitación de manera que tengan mayor cobertura y se aproveche al máximo el interés del profesorado. 
Los resultados obtenidos señalan que la incorporación de las TIC se lo hace para apoyar el proceso de planificación formal y documentación de la clase más no para incorporar las TIC al aprendizaje de los estudiantes. Se determina también que el cambio generacional, determinado por la edad de los docentes, influye en el uso de las TIC.

\section{Bibliografía}

Área, M. (2007). Algunos principios para el desarrollo de buenas prácticas pedagógicas con las TICs en el aula. Comunicación Y Pedagogía: Nuevas Tecnologías Y Recursos Didácticos, 222, 42-47. Retrieved from http://manarea.webs.ull.es/wp-content/uploads/2010/06/CyPbuenaspracticasTIC.pdf

Belloch, C. (2012). Las Tecnologías de la Información y Comunicación en el aprendizaje. Retrieved from http://www.uv.es/bellochc/pedagogia/EVA1.pdf

Boza, Á. Toscano, M., \& Mendez, J. (2009). El impacto de los proyectos TICS en la organización y los procesos de enseñanza-aprendizaje en los centros educativos. Revista de Investigación Educativa, 27(1), 263-289. Retrieved from http://revistas.um.es/rie/article/view/94401/103031

Chiappe, A., Mesa, N., \& Álvarez, C. (2013). Transformaciones en las Concepciones de los Docentes de Educación Secundaria acerca de la Web 2. 0 y su uso en los procesos de enseñanza. Estudios Pedagógicos, 39(2), 55-66. Retrieved from http://mingaonline.uach.cl/scielo.php?script=sci_issuetoc $\&$ pid=0718-

$070520130002 \& \operatorname{lng}=$ es\&nrm $=$ iso

Comisión Económica para América Latina y el Caribe. (2013). Lista de indicadores para el eLAC2015. Retrieved from http://www.cepal.org/es/publicaciones/4052-lista-indicadoreselac2015-version-2013

Instituto Nacional de Estadísticas y Censos. (2015). Tecnologías de la Información y Comunicaciones (TIC'S) 2015. https://doi.org/10.1109/ColombianCC.2013.6637543

Kumari, S., \& D’Souza, F. (2016). Secondary school teachers' digital literacy and use of ICT in teaching and learning, I (I), 141-146. Retrieved from http://ijcrd.dvpublication.com/2016/12/23/secondary-school-teachers-digital-literacy-anduse-of-ict-in-teaching-and-learning/

Ministerio de Educación. (2007). Proyecto emergente de Unidades Educativas del Milenio y Establecimientos Réplica. Retrieved from http://educacion.gob.ec/proyecto-emergente-deunidades-educativas-del-milenio-y-establecimientos-replica/

Ministerio de Educación. (2014). Docentes de Pastaza recibieron computadoras, p. 1. Retrieved from http://educacion.gob.ec/docentes-de-pastaza-recibieron-computadoras/ 
Ministerio de Educación. (2016). Currículo de los niveles de Educación Obligatoria. Retrieved from http://educacion.gob.ec/wp-content/uploads/downloads/2016/08/Curriculov2.pdf

Peñaherrera, M. (2012). Uso de Tic en Escuelas Públicas de Ecuador: Análisis, Reflexiones Y Valoraciones. Edutec. Revista Electrónica de Tecnología Educativa, 40, 1-16. Retrieved from http://www.edutec.es/revista/index.php/edutec-e/article/view/364

Salinas, J. (2008). Innovación educativa y uso de las TIC. (Universidad Internacional de Andalucía, Ed.). $\quad$ Sevilla. $\quad$ Retrieved from http://dialnet.unirioja.es/servlet/articulo?codigo=2906571

United Nations Educational Scientific and Cultural Organization. (2013). Uso de TIC en Educación en América Latina y el Caribe. Análisis regional de la integración de las TIC en la educación y de la aptitud digital (e - readiness). Montreal: Instituto de Estadística.

Valdivieso, T. (2010). Uso de tic en la práctica docente de los maestros de educación básica y bachillerato de la ciudad de Loja. Revista Electrónica de Tecnología Educativa, (33), 113. Retrieved from http://www.edutec.es/revista/index.php/edutec-e/article/view/429

Wengslinky, H. (1998). Does It Compute? The Relationship between Educational Technology and Student Achievement in Mathematics. Princeton. Retrieved from http://files.eric.ed.gov/fulltext/ED425191.pdf 